\title{
The neural networks used in FDM printing study
}

\author{
Adriana Munteanu*, and Dragos-Florin Chitariu \\ Technical University Gheorghe Asachi of Iasi, Department of Machining Tool, Romania
}

\begin{abstract}
Neural networks have aroused a lively interest since 1943 when Warren McCulloch and Walter Pitts proposed a neural network model (a single layer model), that has remained fundamentally structural even today for most neural networks. Problem solving and implicit the study of a system's operating model such as 3D printing involves the association between input data, hypotheses and output data, and neural networks provide the ability to form their own model of solving. The main difference between neural networks and other information processing systems is the ability to learn from interacting with the environment and so improving performance. A correct representation of information, allowing interpretation, prediction, and response to an external stimulus, can allow the network to build a model of the considered process, in the paper case fused deposition modelling (FDM) process.
\end{abstract}

\section{Introduction}

3D printing techniques are used today because products are getting much faster by reducing time and it is possible to obtain nowadays almost any kind of geometry that is intended to be realised. If only 10 years ago it was possible to print just one item, now the capability of the equipment permits the use of over 2000 parts so it is possible to infer where the production of the components will be over 10 years. 3D printing technologies allow the delivery of solutions that are somewhere at the border between processing, digital technologies and the internet, and the beneficiaries discover how easy it is to create and produce almost anything. The biggest benefit is that the user can use a simple interface to get a personalized product, and that's what every customer wants.

The concept of personalized printing is interesting for almost everybody. This is a revolutionary method for creating $3 \mathrm{D}$ models and is great for making fast prototypes. By using the utility of inkjet technology saves time and now, it is create a complete model in a single process using 3D printing [1-2].

The rapid prototyping have different advantages as the capability of the method or of the system to produce functional assemblies with the computer aided design help so that to reduce production time. The development of new materials increases the applicability of this technology.

Paper on FDM [1, 3, 4] shown that process parameters such as layer thickness, part build orientation and other parameters have significantly influence on the part dimensional accuracy, roughness and strength of the realized part. The determination of these

*Corresponding author: adycypmunteanu@yahoo.com 
parameters for part quality improvement, particularly roughness, by using traditional methodologies will be costly and time consuming for the required level of precision. To solve this problem, present study proposes the artificial neural network (ANN) method for modelling the relationship between temperature, speed and layer thickness.

Many studies are made on the influence of various process parameters on mechanical proprieties of the print [5-7]. From these parameters the layer thickness, the raster angle and the air gap influence mostly the elastic performance of the ABS printing.

\section{Experimental researches}

In general, any experiment aims to determine the relationships established between the factors who characterize the phenomenon investigated. Due to the complexity of the phenomena, some aspects have to be considered in this study:

- The impossibility of considering the phenomenon in its entirety, with all its interactions and all the factors that determine it;

- The existence of a natural variability of processes and phenomena as a universal feature of the material world.

- The complexity of technological processes that involves a deeply study of them by taking into account some simplifying assumptions.

One of the main issues of technological process research is the establishment of technological parameters that meet certain requirements regarding the productivity and quality of the obtained parts. These researches must be carried out with as little work as possible and with minimal costs.

The quality and precision of the processed surfaces is a criterion of utmost importance in terms of general efforts to raise the quality of production, especially in the case of final operations.

In order to improve process knowledge regarding the $3 \mathrm{D}$ printing and particular the FDM technology and to provide a viable guide to the feature optimization of the process, one can use a new tool such as artificial neural network. This technique allowed developing multiple-variables, non-linear models that can be integrated into real-time process control plan [2]. So that by means of a free available program DTREG, one may create various models like: classical, single-tree models and also TreeBoost and Decision Tree Forest models, consisting of assemblies of many trees. DTREG also can generate Neural Networks, Support Vector Machine (SVM), Gene Expression Programming/Symbolic Regression, K-Means clustering, GMDH polynomial networks, Discriminant Analysis, Linear Regression and Logistic Regression models [3, 8].

Table 1. Input values versus output values.

\begin{tabular}{|l|l|l|l|l|l|}
\hline \multirow{2}{*}{ No. } & \multicolumn{3}{|c|}{ Inputs } & \multicolumn{2}{c|}{ Output } \\
\cline { 2 - 6 } & $\mathbf{T}\left[{ }^{\circ} \mathrm{C}\right]$ & $\mathbf{V}[\mathrm{mm} / \mathrm{s}]$ & $\mathbf{d}_{\mathbf{s}}[\mathrm{mm}]$ & $\mathrm{Ra}$ & $\mathrm{Ra}^{\mathrm{t}}$ \\
\hline 1. & 240 & 30 & 0.1 & 5.891 & 5,812 \\
\hline 2. & 270 & 30 & 0.1 & 6.195 & 6,25 \\
\hline 3. & 240 & 70 & 0.1 & 6.825 & 6,435 \\
\hline 4. & 270 & 70 & 0.1 & 7.156 & 6,123 \\
\hline 5. & 240 & 30 & 0.2 & 7.972 & 8,052 \\
\hline 6. & 270 & 30 & 0.2 & 8.542 & 8,49 \\
\hline 7. & 240 & 70 & 0.2 & 8.287 & 8,675 \\
\hline 8. & 270 & 70 & 0.2 & 7.329 & 8,363 \\
\hline $\begin{array}{l}\text { Mean } \\
\text { values }\end{array}$ & $\mathrm{M}$ & $\mathrm{M}^{\mathrm{t}}$ & & $\mathbf{7 , 2 7 5}$ & $\mathbf{7 , 2 7 5}$ \\
\hline
\end{tabular}


The quality of the printed parts is appreciated in the present work by the roughness of the surfaces, which represents the assembly of relatively small micron regularity relative to the depth and which forms the relief of the actual surface of a part.

The measurement was realized by using a Mitutoyo type inductive roughness. The roughening measurement conditions and the output parameter are presented in Table 1. For experimental data acquisition it has taken into account, in choosing process input parameters, the capability of the $3 \mathrm{D}$ printer and the preliminary tests made. These preliminary tests have allowed the establishment of the levels of each factor and the consideration of the technological possibilities of $3 \mathrm{D}$ printing (the research was made for a non commercial printer). The technological parameters was considered and varied depending on printer capability, for an ABS material with a filament diameter of $1.75 \mathrm{~mm}$ that has good mechanical properties, with excellent impact strength. In terms of print speed, the printer can operate at speeds between $30-70 \mathrm{~mm} / \mathrm{s}$. The thickness of the deposited layer may vary between $0.05 \mathrm{~mm}$ and $0.3 \mathrm{~mm}$. The printer has a single extruder with a diameter of $0.4 \mathrm{~mm}$ that can withstand temperatures up to $300^{\circ} \mathrm{C}$. The quality of the fused deposition modeling parts depends on a great number of parameters both of the workpiece material and of the process. The parameters chosen was made with Taguchi method so that to reduce the input parameters without losing the precision of the experiment results.
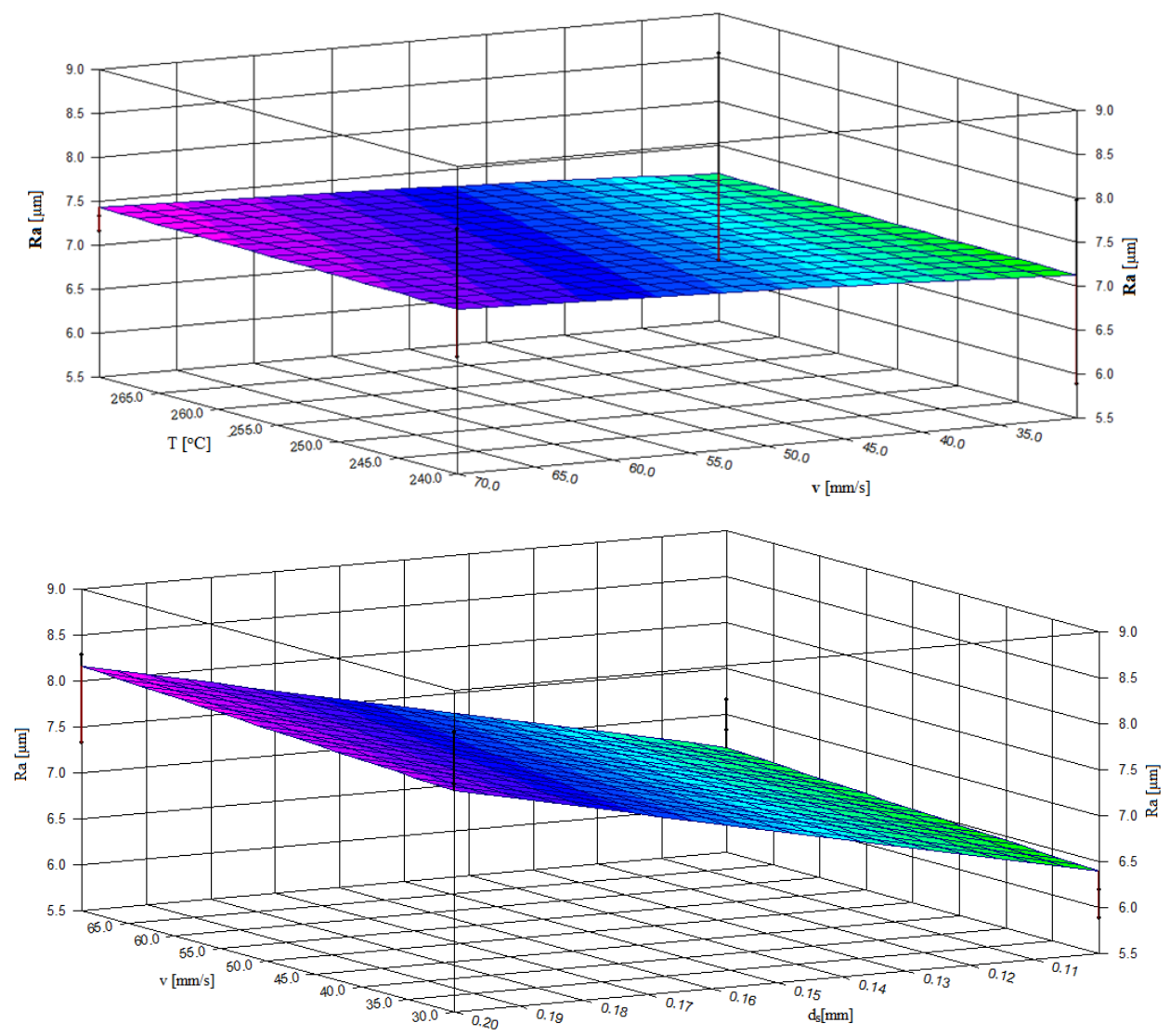


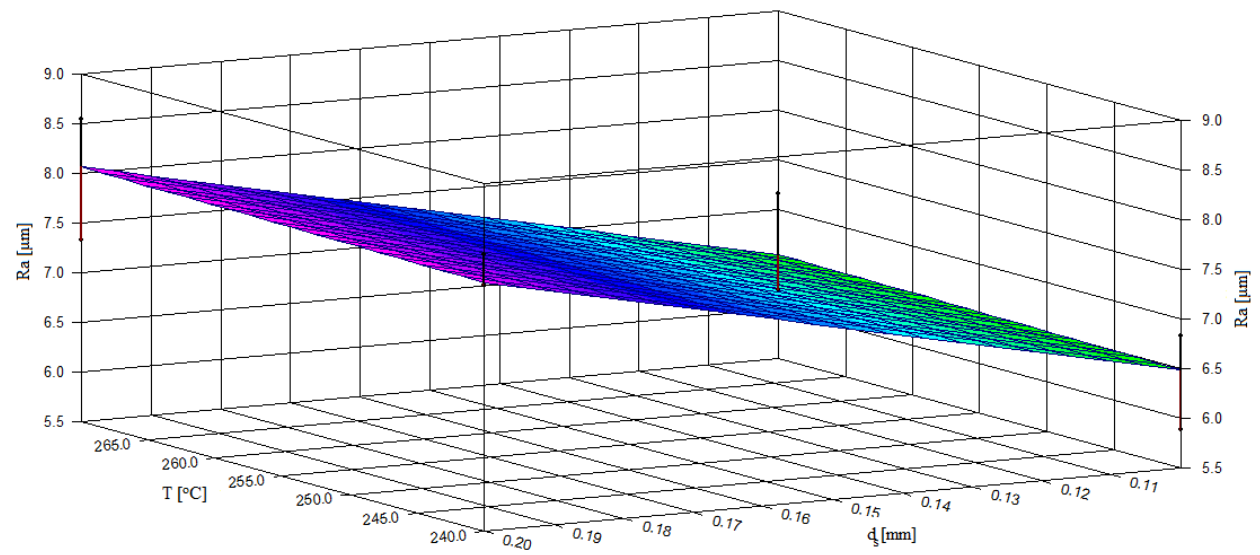

Fig. 1. The variation of the input parameter for Ra parameter.

The variation of the output parameter according to the input parameters can be seen in the graphs of the Figure 1.The graphs show that for this particular case that: the input parameters $d_{s}$ and $v$ act to increase the roughness (positive response of the system for the increase of these factors); the input factor $T$ acts to decrease the roughness of the printed surface when is increasing this factor and the factor $d$ has the greatest influence (the slope being the largest) and acts in the sense of increasing the roughness of the printed surface by the FDM process, is the main factor [8].

The ANN has the ability to learn from examples make them attractive because did not follow a set of rules and learn by underlying rules of given collection of representative examples. For parameter prediction in case of roughness of the printed part the program can predict the value of an output parameter giving enough input parameter information. In order to model this parameter, an architecture that has 3 levels of layers (1 hidden) was used. The neural networks for roughness is a multilayer perceptron neural network type with one hidden layer, with a variable number of units in the input layer and the hidden layer.

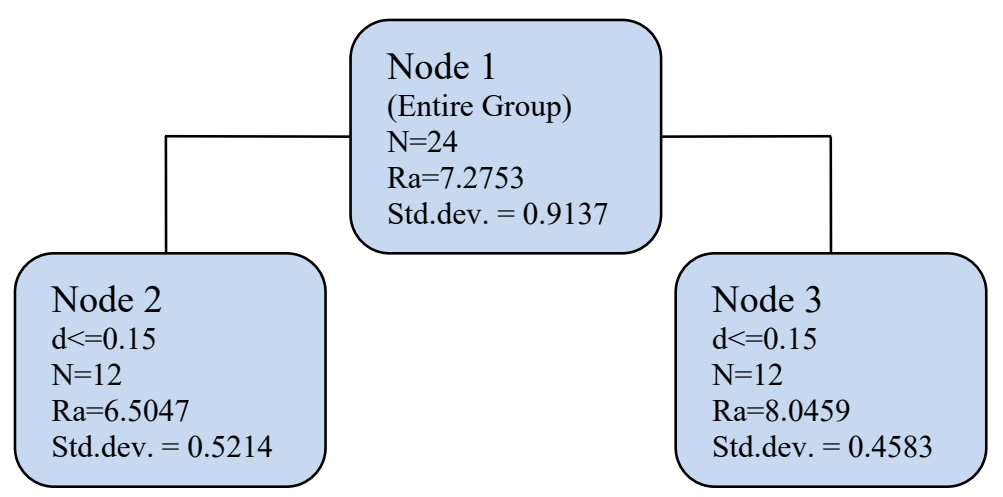

Fig. 2. The main values in nodes.

The number of predictor variables was 3. The neurons found in hidden layer were supposed from 2 to 20 , and it was established at 14 . The main values for the roughness are presented in figure 2 through the single tree models with the main values in nodes. 
A graphic representation of the experimental data obtained versus predicted values for the $R a$ (roughness parameter) is presented in the Figure 3 .

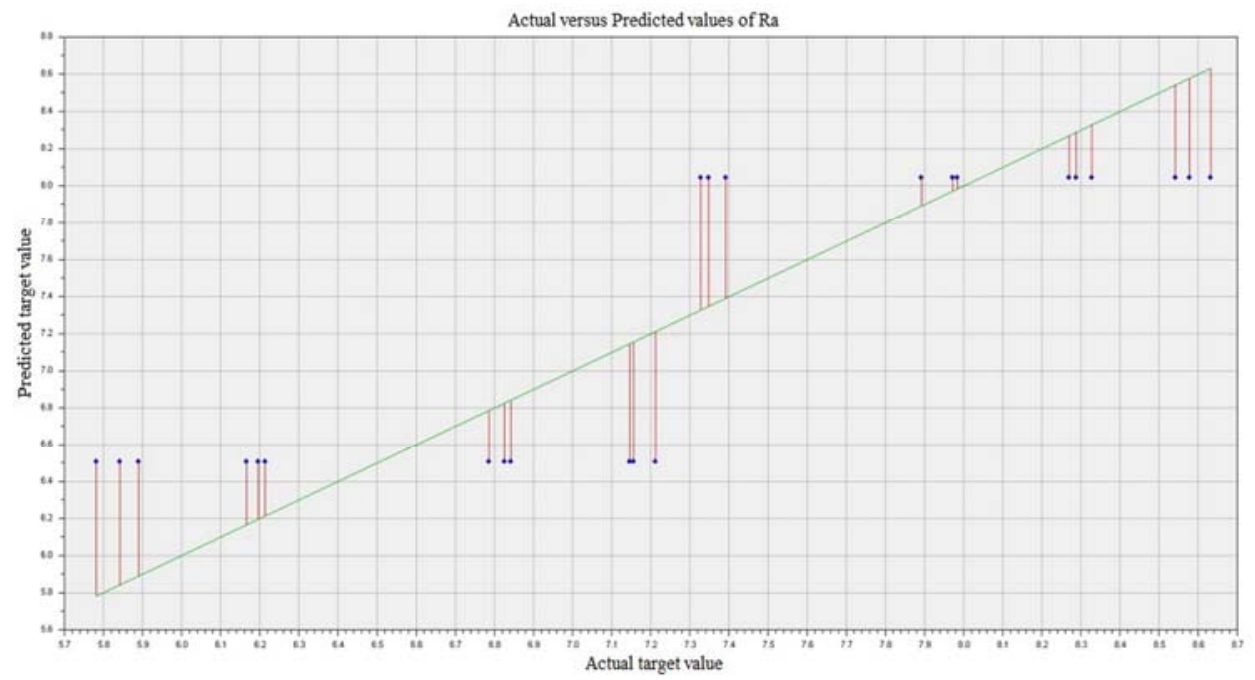

Fig. 3. Actual versus predicted values for Ra.
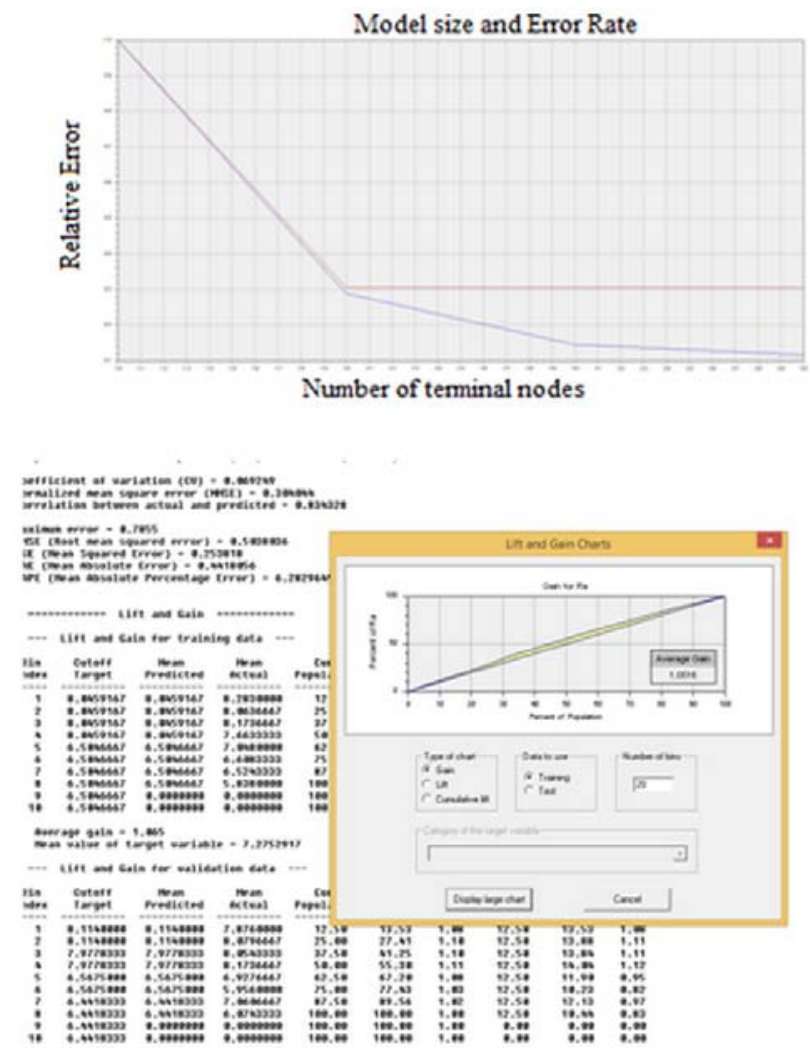

Fig. 4. Model size. 
The Actual versus Predicted chart is available only for building the model in Figure 3. When the predicted value differs from the actual value, the points are offset from the diagonal line, and the vertical distance from the line to the point corresponds to the error (residual).

\section{Conclusions}

The prototype parts solve the necessity of the industrial beneficiaries regarding the products, that must be functional, with a reasonably price and easy to use. This innovative and modern technology (fused deposition modelling) FDM has proven its value assuring functional prototypes of plastic with time, money and manpower saving. The application of artificial neural networks modelling technique allowed the improvement of the process knowledge, and therefore to use this aspect to facilitate process control. The Taguchi method was used for parameters chosen so that to reduce the input parameters number (only three input parameter was considered) without losing the precision of the experiment results.

The model size (graphical representation) emphasize that the output parameter $\mathrm{Ra}$ is closer to the expected output (the superior curve, Figure 4). The main difference between neural networks and other information processing systems is the ability to learn from interacting with the environment and so improving performance. From the results, the prediction of the surface roughness of FDM parts emphasize that the main factor was the layer thickness $\left(d_{s}\right)$. The importance of the layer thickness is $100 \%$ relative to the other two input parameters, the temperature and deposition speed.

The reason of neural networks use is its ability meaning from imprecise data so that to extract patterns and detect trends. In upcoming works the research will also focus on analyzing other parameters and then summing these analyzes to make an optimization of chosen the parameters for the particular printer case.

\section{References}

1. B.H. Lee, J. Abdullah, Z.A. Khan, J Mat. Process. Tech., 169, 54-61 (2005)

2. K. J.Anil, K.M.Mohiuddin, J.Mao, IEEE, (1996)

3. Anoop Kumar Sood, R. K. Ohdar, S. S. Mahapatra, Swarm, Evol. and Mem. Comp., 6466, 396-403 (2010)

4. A.K. Sood, R.K. Ohdar, S.S. Mahapatra, Mater. and Des. 30(10), 4243-4252 (2009)

5. D. Ahn, J.H. Kweon, S. Kwon, J. Song, S. Lee, J. of Mater Process Technol. 209(1516), 5593-5600 (2009)

6. A. Bernarand, A. Fischer, CIRP annals-Man. Tech., 51, 635-52 (2002)

7. L. Novakova - Marcincinova, Manuf. and Ind. Eng., 11, 35-37 (2012)

8. *** P.H. Sherrod, DTREG Predictive modelling software. Available from www.dtreg.com (2018) 